\title{
Antibiotic resistance pattern of Pseudomonas aeruginosa isolated from patients of lower respiratory tract infection
}

\author{
Piyush Tripathi ${ }^{1}$, Gopa Banerjee ${ }^{1 *}$, Shivani Saxena ${ }^{1}$, Mahendra Kumar Gupta ${ }^{1}$, and \\ P. W. Ramteke ${ }^{2}$ \\ ${ }^{1}$ Department of Microbiology, Chhatrapati Shahuji Maharaj Medical University, Lucknow, (U.P.) 226003, India. \\ ${ }^{2}$ Sam Higginbottom Institute of Agriculture, Technology and Sciences, Allahabad, India.
}

Accepted 22 August, 2011

\begin{abstract}
The aim of this study was to determine the antibiotic susceptibility pattern of Pseudomonas aeruginosa from sputum samples of lower respiratory tract infection (LRTI) patients admitted to the Department of Pediatrics and Pulmonary Medicine and also to update clinicians on various antimicrobial alternatives available in treatments. Sputum samples from 298 patients were cultured identified and antibiotic sensitivity was performed by standard methods. Out of the 298 sputum samples from LRTI patients 102 $(34.23 \%)$ patients had established $P$. aeruginosa aetiology. Cefepime and ceftazidime show more resistance to $\boldsymbol{P}$. aeruginosa isolates with resistance 36.27 and $35.30 \%$, respectively. Least resistance was noted for piperacillin/tazobactam and Imipenam that is, 4.90 and $5.88 \%$, respectively. Fifty percent $P$. aeruginosa are intermediate or marginally sensitive to gentamycin. Piperacillin/Tazobactam and Imipenam was found to be the most sensitive drug against $P$. aeruginosa in LRTI patients. Gentamycin resistance is continuously increasing.
\end{abstract}

Key words: Antibiotic susceptibility profile of $P$. aeruginosa, lower respiratory tract infection, multiple antibiotic resistance.

\section{INTRODUCTION}

Lower respiratory tract infections (LRTI) are very common in general practice and comprise of bronchitis and pneumonia (Macfarlane et al., 2001). It is associated with considerable mortality and morbidity worldwide.

Infections of the lower respiratory tract are responsible for $4.4 \%$ of all hospital admissions and $6 \%$ of all general practitioner consultations (Anderson et al., 1993). They also account for 3 to $5 \%$ of deaths in adults, especially over the age of 60 years (Gordon, 1974).

Pseudomonas aeruginosa is a motile gram-negative rod that belongs to the family Pseudomonadaceae. $P$. aeruginosa is widely distributed in narture, but has higher prevalence in hospital environment, as the wards encourage bacterial growth (Hugbo et al., 1992). The

${ }^{*}$ Corresponding author. E-mail: gopa.banerjee31@rediffmail.com. Tel: 0522 - 2257569. characteristic features of $P$. aeruginosa isolates that allows the persistence in hospital is its ability to acquire resistance to variety of antibiotics, withstands physical conditions like temperature, high concentration of salts and antiseptics.

$P$. aeruginosa infections of the lower respiratory tract can range in severity from colonization (without an immunological response) to a severe necrotising bronchopneumonia (Banerjee et al., 2000).

$P$. aeruginosa isolates are naturally resistant to large number of antibiotics that can be acquired during treatment (Bonfiglio et al., 1998) as a result of treatment failure (Ebie et al., 2001). Consequential effect of high resistance pattern is responsible for high mortality rate associated with pseudomonal infections (Samporn et al., 2004).

Antibiotic resistance pattern of $P$. aeruginosa isolates varied with geographical location and hospitals environments. Therefore, chemotherapeutic approach of 


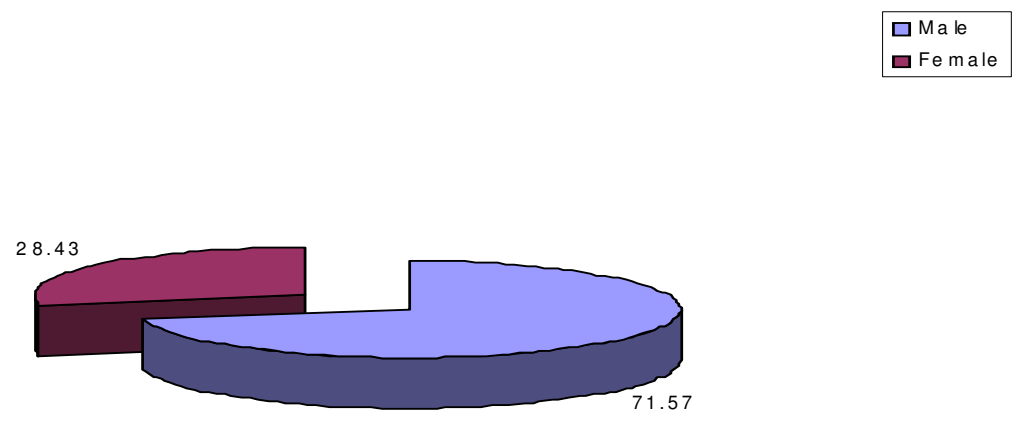

Figure 1. Percentage distribution of lower respiratory tract infections by sex.

pseudomonal infection would depend on peculiarity of the isolates susceptibility pattern, in order to safeguard against treatment. In this context, this study examined the antibiotic resistance of $P$. aeruginosa isolates from sputum in a tertiary care hospital.

\section{MATERIALS AND METHODS}

\section{Study population}

Sputum samples were collected from patients attending to department of Pediatrics and department of pulmonary Medicine of Gandhi Memorial and Associated Hospital of Chhatarpati Shahuji Mharaj Medical University, Lucknow, India.

\section{Sample collection}

The samples were collected and labeled at the Medical Laboratory Unit of the Health Services Department of the University. These samples were analyzed within $30 \mathrm{~min}$ to $1 \mathrm{~h}$ of collection.

\section{Culture, characterization and identification of Pseudomonas from sputum samples}

The samples were streaked on nutrient agar, Mac Conkey agar and blood agar. The plates were incubated at $37^{\circ} \mathrm{C}$ for $24 \mathrm{~h}$ as described by Cheesborough (2002). Isolates obtained after incubition were subcultured using isolation media, that is, Pseudomonas isolation agar. The pure isolates of Pseudomonas were transferred to $1 \%$ nutrient agar slant and stored in the refrigerator at $4 \pm 1^{\circ} \mathrm{C}$. Suspected Pseudomonas species were characterized and identified according to standard bacteriological methods, gram stains and biochemical tests such as oxidase, nitrate reduction, citrate utilization, oxidative fermentation, arginine, and growth at $42^{\circ} \mathrm{C}$.

\section{Antibiotic susceptibility test}

The antibiotic susceptibility patterns of the isolates to common antibiotics used in the hospital were determined using the agar-disk diffusion method on Mueller-Hinton agar described by Ebie et al. (2001) and in the Manual of Antimicrobial Susceptibility Testing (Coyle, 2005). Five discrete colonies were inoculated into $5 \mathrm{ml}$ of sterile nutrient broth and incubated at $37^{\circ} \mathrm{C}$ over night. The broth culture was then diluted 1:10 with a freshly prepared nutrient broth to give a count of approximately 105 colonies per millimeter. An overnight broth culture of each isolate was uniformly spread onto the surface of the Mueller-Hinton plates. A sterile cotton wool was allowed to soak in the broth culture, squeezed by the side of the bottle before streaking over the sensitivity plates and incubated at $37^{\circ} \mathrm{C}$ for $18 \mathrm{~h}$. The appropriate antibiotic multi-discs were aseptically placed on the agar using sterile forceps. The plates were then incubated at $37^{\circ} \mathrm{C}$ for $24 \mathrm{~h}$. The degree of susceptibility of the test isolate to each antibiotic was interpreted as either sensitive (S), marginally sensitive (MS) or resistant (R) by measuring the zone diameter of inhibition. The antibiotic discs contained the following antibiotic concentrations: piperacillin/ tazobactum $110 \mathrm{mcg}$, amikacin $30 \mathrm{mcg}$, ceftazidime $30 \mathrm{mcg}$, ciprofloxacin $5 \mathrm{mcg}$, meropenum $10 \mathrm{mcg}$, imipenum $10 \mathrm{mcg}$, levofloxacin $5 \mathrm{mcg}$, cefepime $30 \mathrm{mcg}$, gentamycin $10 \mathrm{mcg}$. Interpretation of results was done using the zone of inhibition sizes. Zones of inhibition of $\geq 18 \mathrm{~mm}$ were considered sensitive, 13 to 17 $\mathrm{mm}$ intermediate or marginally sensitive and $<13 \mathrm{~mm}$ resistant.

\section{Quality control}

Quality control was performed utilizing strains from the American type culture collection (ATCC), P. aeruginosa ATCC 27853.

\section{MAR index}

The multiple antibiotic resistance MAR index was determined for each isolate by dividing the number of antibiotics to which the isolate is resistant by the total number of antibiotics tested (Krumpernam, 1983).

MAR index $=$ Number of antibiotics isolate is resistant to/ Total number of antibiotics tested

\section{RESULTS}

Sputum specimens were collected from 298 patients with LRTI during a period of one year. Out of these, 102 (34.23\%) patients had an established $P$. aeruginosa aetiology, and of these, $73(71.57 \%)$ were males and 29 (28.43\%) females (Figure 1).

Table 1 summarizes the susceptibility testing results obtained with $P$. aeruginosa. The most active compound against this was piperacillin/tazobactam (89.22\%). The spectrum rank order of the antimicrobial agents against 
Table 1. Antimicrobial susceptibility pattern of Pseudomonas aeruginosa isolates

\begin{tabular}{lccc}
\hline \multirow{2}{*}{ Antibiotics } & \multicolumn{3}{c}{ Susceptibility } \\
\cline { 2 - 4 } & S & MS & R \\
\hline Piperacillin/Tazobactam & $91(89.22 \%)$ & $6(5.88 \%)$ & $5(4.90 \%)$ \\
Amikacin & $84(82.35 \%)$ & $7(6.86 \%)$ & $11(10.79 \%)$ \\
Ceftazidime & $62(60.78 \%)$ & $4(3.92 \%)$ & $36(35.30 \%)$ \\
Ciprofloxacin & $56(54.90 \%)$ & $14(13.73 \%)$ & $32(31.37 \%)$ \\
Meropenam & $67(65.68 \%)$ & $14(13.73 \%)$ & $21(20.59 \%)$ \\
Imipenam & $89(87.26 \%)$ & $7(6.86 \%)$ & $6(5.88 \%)$ \\
Levofloxacin & $41(40.20 \%)$ & $28(27.45 \%)$ & $33(32.35 \%)$ \\
Cefepime & $37(36.27 \%)$ & $28(27.45 \%)$ & $37(36.27 \%)$ \\
Gentamycin & $22(21.57 \%)$ & $51(50.00 \%)$ & $29(28.43 \%)$ \\
\hline
\end{tabular}

$\mathrm{S}=$ sensitive, $\mathrm{R}=$ resistant, $\mathrm{MS}=$ marginally sensitive.

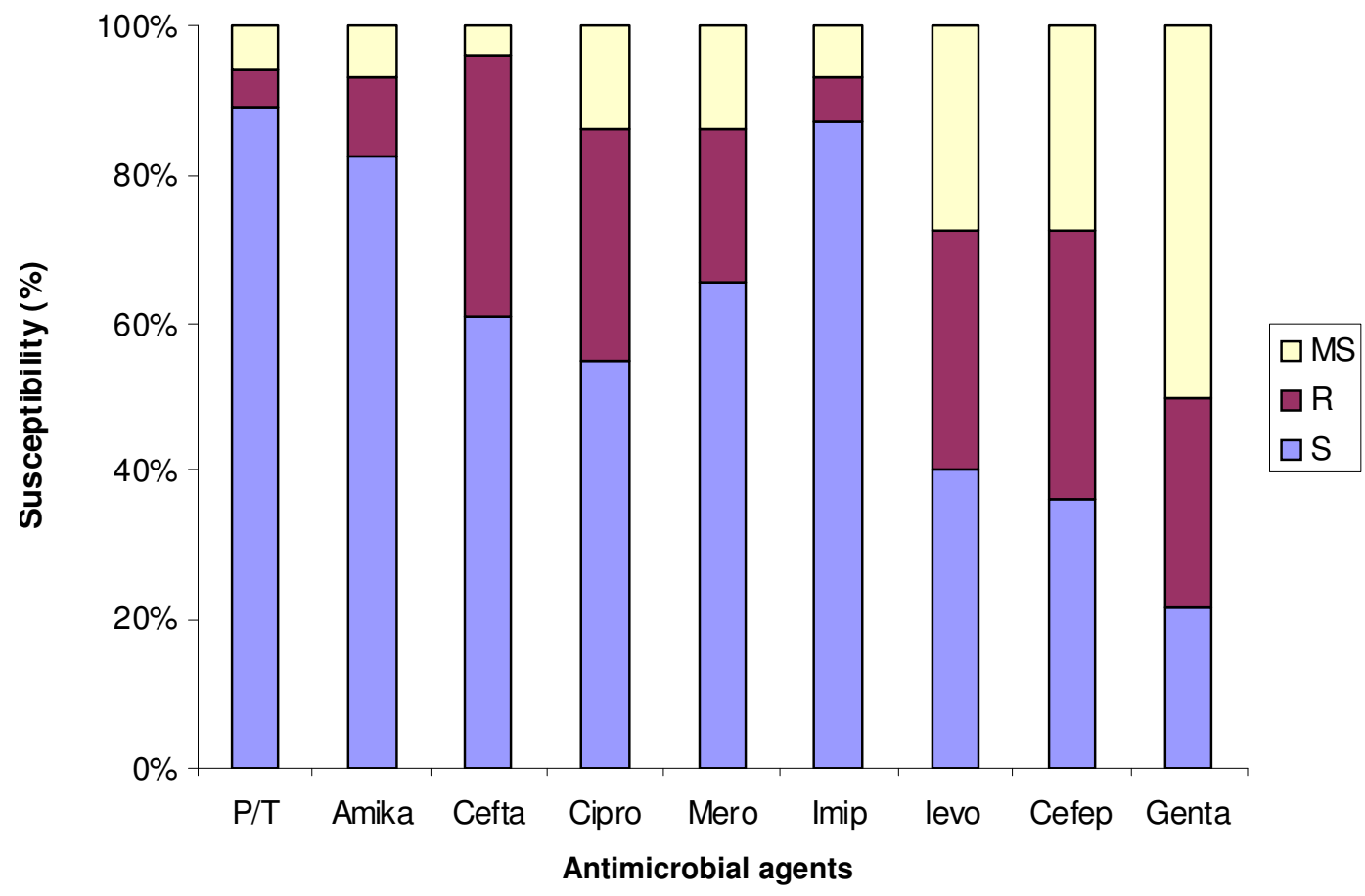

Figure 2. Antimicrobial susceptibility profile of $P$. aeruginosa isolates. $\mathrm{S}=$ sensitive, $\mathrm{R}=$ resistant, $\mathrm{MS}=$ marginally sensitive P/T = piperacillin/tazobactam, Amika = amikacin, Cefta = ceftazidime, Cipro = ciprofloxacin, Mero = meropenam, Imip = imipenam, Levo = levofloxacin, Cefep $=$ cefepime, Genta= gentamycin.

$P$. aeruginosa in terms of percentage of susceptibility was: piperacillin/tazobactam $(89.22 \%)>$ imipenam $(87.26 \%)>$ amikacin $(82.35 \%)>$ meropenam $(65.68 \%)>$ ceftazidime $(60.78 \%)>$ ciprofloxacin $(54.90 \%)>$ levofloxacin $(40.20 \%)>$ cefepime $(36.27 \%)>$ gentamycin (21.57\%).

Cefepime and ceftazidime shows more resistance to $P$. aeruginosa isolates with resistance 36.27 and $35.30 \%$, respectively, followed by levofloxacin (32.35\%) > ciprofloxacin (31.37\%) > gentamycin $(28.43 \%)>$ meropenam (20.59\%).

Figure 2 shows the graphical presentation of antibiotic susceptibility profile of $P$. aeruginosa isolates in which we can clearly see that cefepime and ceftazidime shows more resistance to $P$. aeruginosa and piperacillin/tazobactam and imipenam are more sensitive than all other drugs used. Table 2 shows multiple antibiotic resistance (MAR) index of $P$. aeruginosa isolates which shows that that $73.6 \%$ had MAR index of 0.3 and above. 
Table 2. Multiple antibiotic resistance (MAR) index of Pseudomonas aeruginosa isolates.

\begin{tabular}{ccc}
\hline MAR index & No of isolates & Percentage \\
\hline 0 & 42 & 41.2 \\
0.1 & 16 & 15.7 \\
0.2 & 10 & 9.8 \\
0.3 & 7 & 6.9 \\
0.4 & 9 & 8.8 \\
0.6 & 7 & 6.9 \\
0.7 & 5 & 4.9 \\
0.8 & 5 & 4.9 \\
0.9 & 1 & 0.9 \\
1 & 0 & 0 \\
\hline
\end{tabular}

\section{DISCUSSION}

Considering $4.90 \%$ resistant to piperacillin/tazobactam, piperacillin with the combination of tazobactam is most affective antibiotic against $P$. aeruginosa followed by imipenam and amikacin with resistant 5.88 and $10.79 \%$, respectively.

Resistance to imipenam has been found to be independent of $\beta$-lactamase production and in $P$. aeruginosa has been attributed to diminished expression of certain outer membrane proteins (Buscher et al., 2000 ). More than $80 \%$ of isolates in this study were sensitive to imipenam (87.26\%) and $5.88 \%$ Pseudomonas strains were resistant. Imipenam is a drug that is not readily available in our environment and its cost is also prohibitive.

Antibiotic resistant to amikacin was near $38 \%$ reported by Mohajeri (2003). P. aeruginosa was 96 and 79\% sensitive to amikacin in United state of America (Streit et al., 2004) and Italy (Blandino et al., 2004), respectively.

Cefepime, ceftazidime and levofloxacin are more resistant antibiotics against $P$. aeruginosa, in LRTI patients, with resistant $36.27,35.30$ and $32.35 \%$ consecutively.

Fifty percent $P$. aeruginosa are intermediate or marginally sensitive to gentamycin with $21.57 \%$ sensitive and $28.43 \%$ resistant, which shows that gentamycin resistance is continuously increasing.

Analysis of the MAR index of the Pseudomonas strains showed that $73.6 \%$ had MAR index of 0.3 and above. MAR index higher than 0.2 has been said to be an indication of isolates originating from an environment where antibiotics were often used (Krumpernam, 1983; Paul et al., 1997). The MAR values can however be viewed as an indication of the extent of microbial exposure to antibiotics used within the community.

The increasing resistance to antibiotics by respiratory pathogens has complicated the use of empirical treatment with traditional agents (Guthrie, 2001) and a definitive bacteriological diagnosis and susceptibility testing would, therefore, be required for effective management of LRTI (Anderson et al., 1993).

In view of the grave consequences of drug resistant $P$. aeruginosa in patients, there is need for urgent action to control the situation. It is therefore recommended that routine microscopy, cultures and antibiotic sensitivity test of clinical samples of patients be carried out so as to enhance the administration of drugs for the treatment and management of LRTI.

Because antimicrobial resistance patterns are continually evolving and resistant $P$. aeruginosa undergo progressive antimicrobial resistance, continuously updated data on antimicrobial susceptibility profiles is essential to ensure the provision of safe and effective empiric therapies (Oteo et al., 2002). Moreover, results obtained from this study must be used to implement prevention programs and policy decisions to prevent emergence and spread of antimicrobial resistance (Oteo et al., 2002). According to Hsu et al. (2007) continued surveillance will also serve as an impartial feedback on the efforts of infection control programs for the future. Such surveillance of clinical microbiology isolates is a critical first step toward controlling the growing worldwide threat of antimicrobial drug resistance and WHONET is a useful tool in this respect. More so, further extensive work should be done to ascertain the extent of these consequences of drug resistant $P$. aeruginosa in our environment.

Indeed, the problem of antibiotic resistance is global. This will greatly help to improve all steps towards the prevention and control of drug resistant organisms in our community.

\section{ACKNOWLEDGEMENT}

Authors would like to thank Department of Science and Technology (DST), Government of India for the financial support for this study.

\section{REFERENCES}

Anderson H, Esmail A, Hollowell J, Littlejohns P, Strachen D (1993). Epidemiologically based needs assessment: lower respiratory disease. DHA Project Research Programme, pp. 6-12.

Banerjee D, Stableforth D (2000). The treatment of respiratory pseudomonas infection in cystic fibrosis: What drug and which way? Drugs, 60(5): 1053-1064.

Blandino G, Marchese A, Ardito F, Fadda G, Fontana R Lo Cascio G, Marchetti F, Schito GC, Nicoletti G (2004). Antimicrobial susceptibility profiles of Pseudomonas aeruginosa and Staphylococcus aureus isolated in Italy from patients with hospital-acquired infections. Int. J. Antimicrobe Agents, 24: 515-518.

Bonfiglio G, Laksai Y, Franchino L Amicosante G, Nicoletti G (1998). Mechanism of $\beta$-lactam resistance amongst Pseudomonas aeruginosa isolated in an Italian survey. J. Antimicrobe Chemother., 42: 697-702.

Buscher KH, Cullman W, Dick W, Wendt S, Opferkuch W (2000). Imipenem resistance in $P$. aeruginosa is due to diminished expression of the outer membrane proteins. J. Infect. Dis., 156: 681685. 
Cheesborough M (2002). Medical laboratories manual for tropical countries. Tropical Health Technol. Butterworth London, 2: 479.

Coyle MB (2005). Manual of Antimicrobial Susceptibility Testing. Am Soc. Microbiol. Press, Washington DC, 25: 39.

Ebie MY, Kandakai-Olukemi YT, Ayanbadejo J, Tanyigna KB (2001). Urinary Tract Infections in a Nigerian Military Hospital. Nig. J. Microbiol., 15(1): 31-37.

Gordon M (1974). Editorial. Antibiotics and respiratory illness. Br. Med. J., 3: 1.

Guthrie R (2001). Community-acquired lower respiratory tract infections: Etiol. Treatment Chest, 20: 2021-2034.

Hsu LY, Tan TY, Jureen R, Koh TH, Krishnan P, Lin RTP, Tee NWS, Tambyah PA (2007). Antimicrobial drug resistance in Singapore hospitals. Emerg. Infect. Dis., 13(12): 1944-1947.

Hugbo PG, Olurinola PF (1992). Resistance of Pseudomonas aeruginosa to antimicrobial agents: Implications in medicine and pharmacy. Nig. J. Pharm. Sci., 4: 1-10.

Krumpernam PH (1983). Multiple antibiotic resistance indexing Escherichia coli to identify risk sources of faecal contamination of foods. Appl. Environ. Microbiol., 46: 165-170.

Macfarlane J, Holmes W, Gard P, Macfarlane R, Rose D, Weston V, Leinonen M, Saikku P, Myint S (2001). Prospective study of the incidence, aetiology and outcome of adult lower respiratory tract illness in the community. Thorax, 56(2): 109-114.
Mohajeri P (2003). Antibacterial resistance and sensitivity pattern in Pseudomonas aeruginosa isolated from various clinical samples of patients referred to Kermansha Hospitals. Behbound Res. J., 7: 1120.

Oteo J, Campos J, Baquero F (2002). Antibiotic resistance in 1962 invasive isolates of Escherichia coli in 27 Spanish hospitals participating in the European Antimicrobial Resistance Surveillance System. J. Antimicrob. Chemother., 50: 945-952.

Paul S, Bezbarauh RL, Roy MK, Ghosh AC (2002). Multiple antibiotic resistance (MAR) index and its reversion in Pseudomonas aeruginosa. Lett. Appl. Microbiol., 24: 169-171.

Samporn S, Chuntima T, Thitiya Y, Chertask D (2004). Prevalence and antimicrobial susceptibility of Pseudomonas aeruginosa mucoid and non-mucoid type. Southeast Asian J. Trop. Med. Publ. Health, 35: 893-894.

Streit JM, Jones RN, Sader HS, Fritsche TR (2004). Assessment of pathogen occurrences and resistance profiles among infected patients in the intensive care unit: Repot from the sentry antimicrobial surveillance program. Int. J. Antimicrobe Agents, 24: 111-118. 